\title{
PDF of Velocity Fluctuation in Turbulence by a Statistics based on Generalized Entropy
}

\author{
Toshihico Arimitsu ${ }^{\mathrm{a}}$ and Naoko Arimitsu ${ }^{\mathrm{b}}$ \\ ${ }^{a}$ Institute of Physics, University of Tsukuba, Ibaraki 305-8571, Japan \\ ${ }^{\mathrm{b}}$ Graduate School of EIS, Yokohama Nat'l. University, Yokohama 240-8501, Japan
}

\begin{abstract}
An analytical formula for the probability density function (PDF) of the velocity fluctuation in fully-developed turbulence is derived, non-perturbatively, by assuming that its underlying statistics is the one based on the generalized measures of entropy, the Rényi entropy or the Tsallis entropy. The parameters appearing in the PDF, including the index $q$ in the generalized measures, are determined self-consistently with the help of the observed value $\mu$ of the intermittency exponent. The derived PDF explains quite well the experimentally observed density functions.
\end{abstract}

Key words: generalized statistics, multifractal, velocity fluctuation, turbulence, intermittency

PACS: 47.27.-i, 47.53.+n, 47.52.+j, 05.90.+m

In this paper, we analyze the probability density function (PDF) of the velocity fluctuations in turbulence measured in the experiments conducted by Lewis and Swinney [1] and by Gotoh et al. [2] in terms of a generalized statistics constructed on Rényi's [3] or on Tsallis' entropy [4,5]. The Rényi entropy has an information theoretical basis, and has the extensive characteristics as the usual thermodynamical entropy does. On the other hand, the Tsallis entropy is non-extensive, and therefore it provides us with one of the attractive trials for generalized statistical mechanics which deals with, for example, systems with long range correlations. Note that the distribution functions which give an extremum of each entropy have a common structure (see (5) for the present system) in spite of different characteristics of these entropies. The investigation of turbulence based on the generalized statistics was set about by the present authors [6] with an investigation of the p-model $[7,8]$. Then, the treatment was sublimated by a self-consistent analysis following the usual procedure of ensembles in statistical mechanics, where it was shown that the derived analytical expression for the scaling exponents of the velocity structure function explains quite well the experimentally observed exponents [9-12]. It has been 
revealed also that, within the same line of the statistical mechanical treatment, the PDF of the velocity fluctuations can be derived analytically $[12,13]$. An input for the ensemble theoretical method is the experimentally measured intermittency exponent. The parameters appearing in the ensemble theory are determined self-consistently with the help of the physically meaningful equations, i.e., the conservation of energy, the definition of the intermittency exponent and the scaling relation relevant to the analysis by statistics based on the generalized entropy [9-13]. The present ensemble theoretical method rests on the following aspects: 1) the fully developed turbulence is the situation where the kinematic viscosity can be neglected compared with the effect of the turbulent viscosity that is a cause of intermittency; 2) in this situation, one can find an invariance of the Navier-Stokes equation in certain scale transformation which contains an arbitrary exponent that measures the degrees of singularity in velocity gradient; 3) for the distribution of the exponent, the statistics based on the generalized entropies is adopted, which was shown [1013] to be a generalization of the statistics in the log-normal model [14-16]. The statistical mechanical procedure based on the Boltzmann-Gibbs entropy gives the results of the log-normal model, whereas the one based on the generalized entropies provides us with the above mentioned new results [12].

The basic equation describing fully developed turbulence is the Navier-Stokes equation $\partial \vec{u} / \partial t+(\vec{u} \cdot \vec{\nabla}) \vec{u}=-\vec{\nabla}(p / \rho)+\nu \nabla^{2} \vec{u}$ of an incompressible fluid, where $\rho, p$ and $\nu$ represent, respectively, the mass density, the pressure and the kinematic viscosity. Our main interest is the correlation of measured time series of the streamwise velocity component $u$, say $x$-component of the fluid velocity field $\vec{u}$. Within Taylor's frozen flow hypothesis, the quantity of our interest reduces to $\delta u(r)=|u(x+r)-u(x)|$ representing the spatial difference of the component $u$. Then, the Reynolds number Re of the system is given by $\operatorname{Re}=\delta u_{0} \ell_{0} / \nu=\left(\ell_{0} / \ell_{\mathrm{K}}\right)^{4 / 3}$. Here, we used $\delta u_{0}=\left(\epsilon \ell_{0}\right)^{1 / 3}$ with the Kolmogorov scale $[17]: \ell_{\mathrm{K}}=\left(\nu^{3} / \epsilon\right)^{1 / 4}(=\eta)$, and the energy input rate $\epsilon$ to the largest eddies of size $\ell_{0}$. Here, we introduced the notation $\delta u_{n}=\delta u\left(\ell_{n}\right)$ representing the velocity difference across a distance $r \sim \ell_{n}$.

For high Reynolds number limit Re $\gg 1$, or for the situation where the effect of the term containing the kinematic viscosity in the Navier-Stokes equation can be effectively neglected, we see that the equation has an invariance under the scale transformation [8]: $\vec{r} \rightarrow \vec{r}^{\prime}, \vec{u} \rightarrow \vec{u}^{\prime}, t \rightarrow t^{\prime}$ and $(p / \rho) \rightarrow(p / \rho)^{\prime}$ with $\vec{r}^{\prime}=\lambda \vec{r}, \vec{u}^{\prime}=\lambda^{\alpha / 3} \vec{u}, t^{\prime}=\lambda^{1-\alpha / 3} t,(p / \rho)^{\prime}=\lambda^{2 \alpha / 3}(p / \rho)$. Here, $\alpha$ is an arbitrary real quantity. Then, we have

$$
\delta u_{n} / \delta u_{0}=\left(\ell_{n} / \ell_{0}\right)^{\alpha / 3}, \quad \epsilon_{n} / \epsilon=\left(\ell_{n} / \ell_{0}\right)^{\alpha-1}
$$

where $\epsilon_{n}$ represents the rate of transfer of energy per unit mass from eddies of size $\ell_{n}$ to eddies of size $\ell_{n+1}$. We have put $\epsilon_{0}=\epsilon$. The first equation in (1) leads to a singularity in the velocity gradient $[18]|\partial u(x) / \partial x|=\lim _{\ell_{n} \rightarrow 0} \mid u\left(x+\ell_{n}\right)-$ 
$u(x) \mid / \ell_{n}=\lim _{\ell_{n} \rightarrow 0} \delta u_{n} / \ell_{n}$ for $\alpha<3$, since $\delta u_{n} / \ell_{n}^{\alpha / 3}=$ constant. The degrees of the singularity are specified by the exponent $\alpha$. The singularities in velocity gradient fill the physical space of dimension $d$ with a fractal dimension $F(\alpha)$. Then, the probability $P(\alpha) d \alpha$ to find a point to be singular is specified by the distribution function of singularities $P(\alpha) \propto \delta_{n}^{d-F(\alpha)}$, which leads through the second relation in (1) that the energy transfer rate $\epsilon_{n}$ is a fluctuating quantity in space. The conservation of energy in the energy-transfer cascade requires that

$$
\left\langle\epsilon_{n}\right\rangle=\epsilon
$$

where $\langle\cdots\rangle$ is defined by $\langle\cdots\rangle=\int d \alpha \cdots P(\alpha)$. The intermittency observed in the systems of fully-developed turbulence is attributed to the distribution of the singularities in each different size $\ell_{n}=\ell_{0} \delta_{n}(n=0,1,2, \cdots)$ of constituting eddies. Here, $\ell_{n}$ represents the diameter of the $n$th eddy, and is determined by the number of steps $n$ of the cascade [19]. It is assumed by putting $\delta_{n}=\delta^{-n}$ $(\delta=2)$ that, at each step of the cascade, eddies break into $\delta$ pieces with $1 / \delta$ diameter. The definition of the intermittency exponent $\mu$ is given by

$$
\left\langle\epsilon_{n}^{2}\right\rangle=\epsilon^{2} \delta_{n}^{-\mu}
$$

It is convenient to introduce multifractal spectrum $f(\alpha)$ by $f(\alpha)=F(\alpha)-$ $(d-1)$ which gives us $P(\alpha) \propto \delta_{n}^{1-f(\alpha)}$.

Regarding an eddy as a cluster having the same size as the eddy [6,12], we can derive the scaling relation $[9-12]$

$$
1 /(1-q)=1 / \alpha_{-}-1 / \alpha_{+}
$$

where $\alpha_{ \pm}$are defined by $f\left(\alpha_{ \pm}\right)=0$. This scaling relation is a manifestation of the mixing property. Note that (4) is a generalization of the scaling relation derived first in $[20,21]$ to the case where the multifractal spectrum $f(\alpha)$ has negative values [22].

Let us find out an appropriate distribution function $P^{(n)}(\alpha) \propto\left[P^{(1)}(\alpha)\right]^{n}$ of the degrees of singularity $\alpha$ at the $n$th step of the cascade. Here, we are assuming the independence of each step. In order to discover $P^{(1)}(\alpha)$, we will take an extremum of the measure of an appropriate generalized entropy with the two constraints, i.e., the conservation of probability: $\int d \alpha P(\alpha)=$ constant, and the fixing of the $q$-variance: $\sigma_{q}^{2}=\left\langle\left(\alpha-\alpha_{0}\right)^{2}\right\rangle_{q}=\left(\int d \alpha P(\alpha)^{q}\left(\alpha-\alpha_{0}\right)^{2}\right) / \int d \alpha P(\alpha)^{q}$. By adopting the measure of the Rényi entropy [3] or of the Tsallis-HavrdaCharvat (THC) entropy $[4,23]$ as an appropriate one, we have the distribution 
function of singularities in the form [9-13]

$$
P^{(n)}(\alpha) d \alpha=\left(Z^{(n)}\right)^{-1}\left[1-\left(\alpha-\alpha_{0}\right)^{2} /(\Delta \alpha)^{2}\right]^{n /(1-q)} d \alpha
$$

with $\Delta \alpha=(2 X /[(1-q) \ln 2])^{1 / 2}$ and an appropriate partition function $Z^{(n)}$. Note that since the Rényi entropy and the THC entropy reduce to the BoltzmannGibbs entropy in the limit $q \rightarrow 1$, the distribution function (5) reduces to the Boltzmann-Gibbs distribution function in that limit. The multifractal spectrum corresponding to the distribution function of singularities is given by [9$13]$

$$
f(\alpha)=1+(1-q)^{-1} \log _{2}\left[1-\left(\alpha-\alpha_{0}\right)^{2} /(\Delta \alpha)^{2}\right]
$$

where we put $f\left(\alpha_{0}\right)=1$, since the fractal dimension of the multifractal sets is one for the present experimental setup of the system. Note that the multifractal spectrum does not depend on the number $n$ of steps in the cascade, which is a manifestation of the assumption that each step is independent.

The mass exponent $\tau(\bar{q})$ is defined by $\delta_{n}^{-d}\left\langle\left(\epsilon_{n} \ell_{n}^{d}\right)^{\bar{q}}\right\rangle=\left(\epsilon \ell_{0}^{d}\right)^{\bar{q}} \delta_{n}^{-\tau(\bar{q})+(\bar{q}-1)(d-1)}$ where $\epsilon_{n} \ell_{n}^{d}$ is the total dissipation in volume $\ell_{n}^{d}$, and the factor $\delta_{n}^{-d}$ is the number of boxes of volume $\ell_{n}^{d}$ in the region of volume $\ell_{0}^{d}$. $\square$ Substituting $P(\alpha) \propto$ $\delta_{n}^{1-f(\alpha)}$ into the definition, we have $\delta_{n}^{-\tau(\bar{q})}=\delta_{n}^{-d} \int_{\alpha_{\min }}^{\alpha_{\max }} d \alpha^{\prime} P\left(\alpha^{\prime}\right) \delta_{n}^{\bar{q}\left(\alpha^{\prime}-1\right)} \propto$ $\delta_{n}^{\bar{q} \alpha_{\bar{q}}-f\left(\alpha_{\bar{q}}\right)}$ by estimating the integral in the limit $\delta_{n} \ll 1$, where $\alpha_{\bar{q}}$ is obtained by solving

$$
\bar{q}=d f(\alpha) / d \alpha
$$

Then, comparing the exponents of $\delta_{n}$ in the estimate of the integral, we know that the relation between the multifractal spectrum $f(\alpha)$ and the mass exponent $\tau(\bar{q})$ is given by

$$
f(\alpha)=\bar{q} \alpha+\tau(\bar{q})
$$

with $\alpha$ being determined by (7). From (7) and (8), we can derive

$$
\alpha=-d \tau(\bar{q}) / d \bar{q}
$$

Note that (7), (8) and (9) constitute a kind of Legendre transformation between the mass exponent $\tau(\bar{q})$ and the multifractal spectrum $f(\alpha)$. Equation (7) is solved, analytically, to give us $\alpha_{\bar{q}}=\alpha_{0}-2 \bar{q} X /\left(1+\sqrt{C_{\bar{q}}}\right)$ with

$\overline{1}$ This definition is a bit different from the one in [6,9] for general $d$. 
$C_{\bar{q}}=1+2 \bar{q}^{2}(1-q) X \ln 2$. Here, we chose the solution which is finite for $q \rightarrow 1$. Then, $\alpha_{\max }=\alpha(\bar{q}=-\infty)$ and $\alpha_{\min }=\alpha(\bar{q}=+\infty)$ are given by $\alpha_{\max }-\alpha_{0}=\alpha_{0}-\alpha_{\min }=\Delta \alpha$. We now know that $\sigma_{q}^{2}=(\Delta \alpha)^{2}(1-q) /(3-q)$. The conditions (2) and (3) can be written, respectively, in terms of the mass exponent as $\tau(1)=0$ and $\mu=1+\tau(2)$.

The three quantities $\alpha_{0}, X$ and $q$ are determined by the three independent equations (2), (3) and (4), once the value of the intermittency exponent $\mu$ is given. The scaling relation (4) can be solved, by substituting $\alpha_{ \pm}=\alpha_{0} \pm \sqrt{2 b X}$ satisfying $f_{\mathrm{T}}\left(\alpha_{ \pm}\right)=0$, in the form $\sqrt{2 X}=\left[\left(\alpha_{0}^{2}+(1-q)^{2}\right)^{1 / 2}-(1-q)\right] / \sqrt{b}$, or $\alpha_{0}=[2 b X+2(1-q) \sqrt{2 b X}]^{1 / 2}$, with $b=\left(1-2^{-(1-q)}\right) /[(1-q) \ln 2]$.

Let us now derive the PDF for the velocity fluctuation $\Pi^{(n)}\left(x_{n}\right)$ with $-\infty<$ $x_{n}<\infty$, where $\left|x_{n}\right|=\delta u_{n} / \delta u_{0}$. It may be reasonable to assume that the probability $\Pi^{(n)}\left(x_{n}\right) d x_{n}$ to find the scaled velocity fluctuation $x_{n}$ in the range $x_{n} \sim x_{n}+d x_{n}$ can be divided into two parts [13]:

$$
\Pi^{(n)}\left(x_{n}\right) d x_{n}=\Pi_{\mathrm{N}}^{(n)}\left(x_{n}\right) d x_{n}+\Pi_{\mathrm{S}}^{(n)}\left(\left|x_{n}\right|\right) d x_{n} .
$$

The first term $\Pi_{\mathrm{N}}^{(n)}\left(x_{n}\right)$, assumed to come from thermal or dissipative fluctuation caused by the kinematic viscosity, will be considered later in this paper after estimating the moments of the velocity fluctuation. The second term $\Pi_{\mathrm{S}}^{(n)}\left(\left|x_{n}\right|\right)$ stems from the multifractal distribution of singularities in the velocity gradient given in (5), i.e., $\Pi_{\mathrm{S}}^{(n)}\left(\left|x_{n}\right|\right) d x_{n}=P^{(n)}(\alpha) d \alpha$. This contribution may be related to the fluctuations caused by the turbulent viscosity. The condition $\int_{-\infty}^{\infty} d x_{n} \Pi^{(n)}\left(x_{n}\right)=1$ determines the normalization factor $Z^{(n)}$ here. For this singular part, the relation between $\left|x_{n}\right|$ and $\alpha$ is given by (1), i.e., $\left|x_{n}\right|=\delta_{n}^{\alpha / 3}$, and, therefore, we have an explicit expression of $\Pi_{\mathrm{S}}^{(n)}\left(\left|x_{n}\right|\right)$ in the form $[12,13]$

$$
\Pi_{\mathrm{S}}^{(n)}\left(\left|x_{n}\right|\right) d x_{n}=\left[3 /\left(Z^{(n)}\left|\ln \delta_{n}\right|\right)\right] G^{(n)}\left(\left|x_{n}\right|\right) d x_{n}
$$

with $G^{(n)}(x)=x^{-1}\left[1-\left(\alpha(x)-\alpha_{0}\right)^{2} /(\Delta \alpha)^{2}\right]^{n /(1-q)}$ and $\alpha(x)=3 \ln x / \ln \delta_{n}$. Note that the singular part of the PDF has a dependence on $\ln \delta u_{n}$.

The $m$ th moment can be calculated as [13] $\left\langle\left\langle\left|x_{n}\right|^{m}\right\rangle\right\rangle=\int_{-\infty}^{\infty} d x_{n}\left|x_{n}\right|^{m} \Pi^{(n)}\left(x_{n}\right)=$ $2 \gamma_{m}^{(n)}+\left(1-2 \gamma_{0}^{(n)}\right) a_{m} \delta_{n}^{\zeta_{m}}$ with $2 \gamma_{m}^{(n)}=\int_{-\infty}^{\infty} d x_{n}\left|x_{n}\right|^{m} \Pi_{\mathrm{N}}^{(n)}\left(x_{n}\right)$ and $a_{3 \bar{q}}=$ $\left(2 /\left[\sqrt{C_{\bar{q}}}\left(1+\sqrt{C_{\bar{q}}}\right)\right]\right)^{1 / 2}$. Here, $\zeta_{m}=\alpha_{0} m / 3-2 X m^{2} /\left[9\left(1+\sqrt{C_{m / 3}}\right)\right]-[1-$ $\left.\log _{2}\left(1+\sqrt{C_{m / 3}}\right)\right] /(1-q)$ is the so-called scaling exponents of velocity structure function, whose expression was derived first in [9-13].

In the following, we will derive the symmetric part of the PDF of the velocity difference. The origin of the asymmetry in the PDF may be attributed to 


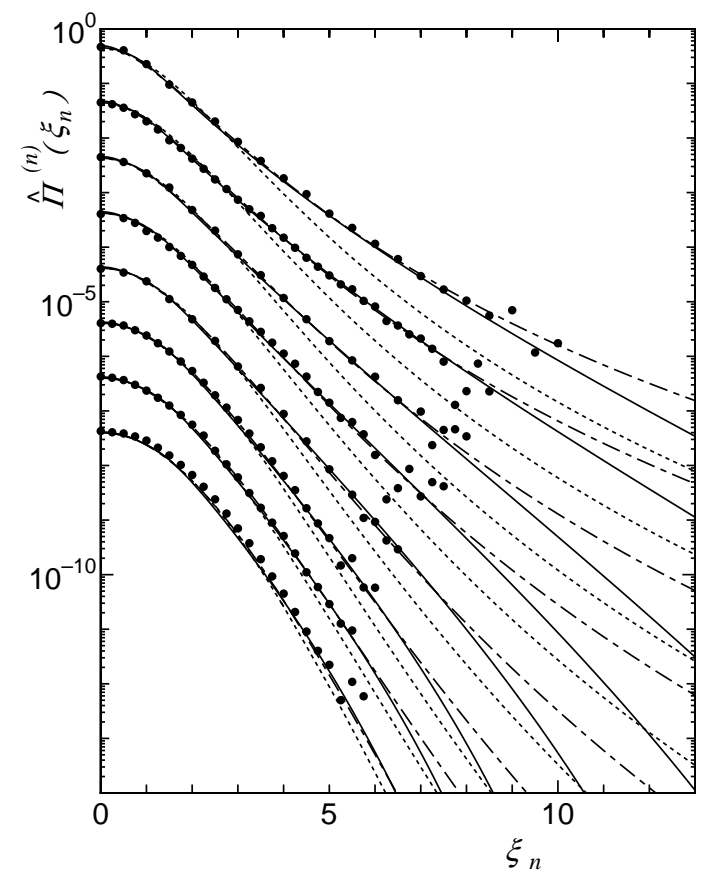

Fig. 1. Experimentally measured PDF of the velocity fluctuations by Lewis and Swinney [1] for $R_{\lambda}=262(\operatorname{Re}=540000)$ and $\mu=0.28$ are compared with the present theoretical results $\hat{\Pi}^{(n)}\left(\xi_{n}\right)$ given by (13) and (14). Closed circles are the symmetrized points obtained by taking averages of the left and the right hand sides of measured PDF. For the experimental data, the distances $r / \eta=\ell_{n} / \eta$ are, from top to bottom: 11.6, 23.1, 46.2, $92.5208,399,830$ and 1440. Solid lines represent the curves given by the present theory with the self-consistently determined parameters $\alpha_{0}=1.162, X=0.334$ and $q=0.471$. For the present theoretical curves (13) and (14), the number of steps in the cascade $n$ are, from top to bottom: 14, 13, 11, 10, 9.0, 8.0, 7.5 and 7.0. Dotted lines are the curves given by directly adjusting the PDF (15) for $\theta=1$, whereas dotted-dashed lines are the ones for $\theta=2-q^{\prime}$. For these adjusted PDF's, the indexes $q^{\prime}$ 's depend on the distances $r / \eta$, and the adjusted values of $q^{\prime}$ are, from top to bottom: 1.168, 1.150, 1.124, 1.105, 1.084, 1.065, 1.055 and 1.038. For better visibility, each PDF is shifted by -1 unit along the vertical axis.

dissipative evolution of eddies or to experimental setup and situations. Let us introduce new variable $\xi_{n}=x_{n} /\left\langle\left\langle x_{n}^{2}\right\rangle\right\rangle^{1 / 2}$ scaled by the variance of the velocity fluctuation, and introduce the probability density function $\hat{\Pi}_{\mathrm{S}}^{(n)}\left(\xi_{n}\right)$ for the singular part with this new variable through the relation $\hat{\Pi}_{\mathrm{S}}^{(n)}\left(\left|\xi_{n}\right|\right) d \xi_{n}=$ $\Pi_{\mathrm{S}}^{(n)}\left(\left|x_{n}\right|\right) d x_{n}$. It is a symmetric function satisfying $\hat{\Pi}_{\mathrm{S}}^{(n)}\left(\xi_{n}\right)=\hat{\Pi}_{\mathrm{S}}^{(n)}\left(-\xi_{n}\right)$. The relation between the new variable and $\alpha$ is given by $\left|\xi_{n}\right|=\delta u_{n} /\left\langle\delta u_{n}^{2}\right\rangle^{1 / 2}=$ $\bar{\xi}_{n} \delta_{n}^{\alpha / 3-\zeta_{2} / 2}$ with $\bar{\xi}_{n}=\left[2 \gamma_{2}^{(n)} \delta_{n}^{-\zeta_{2}}+\left(1-2 \gamma_{0}^{(n)}\right) a_{2}\right]^{-1 / 2}$ and the explicit form of $\hat{\Pi}_{\mathrm{S}}^{(n)}\left(\left|\xi_{n}\right|\right)$ in terms of $\alpha$ reduces to

$$
\hat{\Pi}_{\mathrm{S}}^{(n)}\left(\left|\xi_{n}\right|\right)=\bar{\Pi}_{\mathrm{S}}^{(n)} \delta_{n}^{\zeta_{2} / 2-\alpha / 3+1-f(\alpha)}
$$


with $\bar{\Pi}_{\mathrm{S}}^{(n)}=3\left(1-2 \gamma_{0}^{(n)}\right) /\left(2 \bar{\xi}_{n} \sqrt{2 \pi X\left|\ln \delta_{n}\right|}\right)$. We see from (12) that when the condition $\zeta_{2} / 2-\alpha / 3+1-f(\alpha)=0$ is satisfied, the strong $n$-dependence of $\hat{\Pi}_{\mathrm{S}}^{(n)}\left(\xi_{n}\right)$ may disappear. Corresponding to the two solutions $\alpha^{*}$ and $\alpha^{\dagger}$ satisfying $\alpha^{*}<\alpha^{\dagger}$ for this condition, we have $\xi_{n}^{\dagger}=\bar{\xi}_{n} \delta_{n}^{\alpha^{\dagger} / 3-\zeta_{2} / 2}$ and $\xi_{n}^{*}=$ $\bar{\xi}_{n} \delta_{n}^{\alpha^{*} / 3-\zeta_{2} / 2}$ satisfying $0<\xi^{\dagger}<\xi^{*}$.

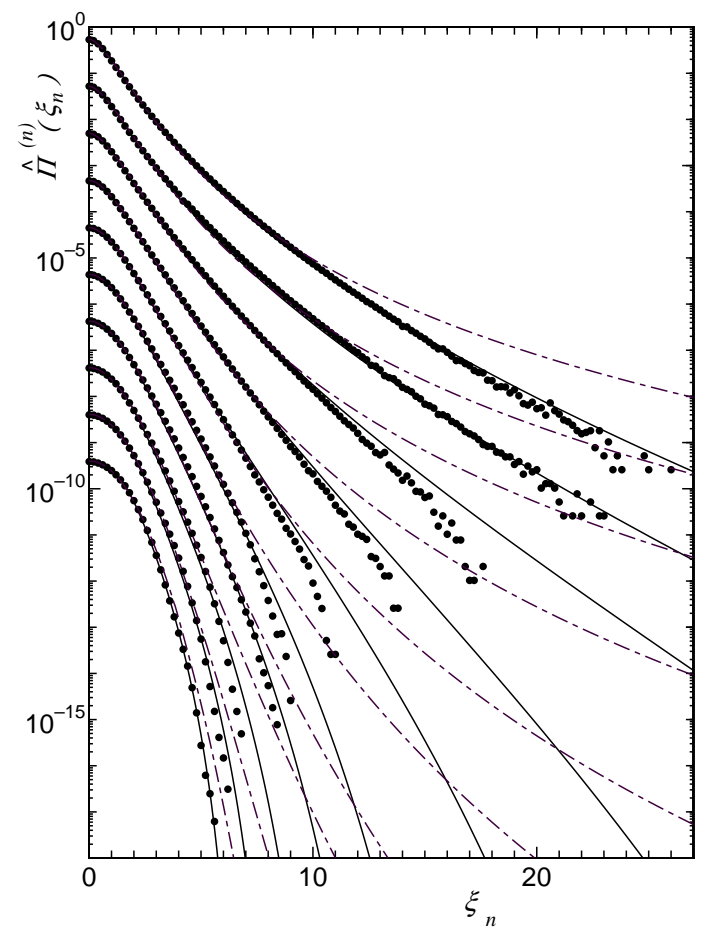

Fig. 2. Experimentally measured PDF of the velocity fluctuations by Gotoh et al. [2] for $R_{\lambda}=381$ and $\mu=0.25$ are compared with the present theoretical results $\hat{\Pi}^{(n)}\left(\xi_{n}\right)$ given by (13) and (14). Closed circles are the symmetrized points obtained by taking averages of the left and the right hand sides of measured PDF. For the experimental data, the distances $r / \eta=\ell_{n} / \eta$ are, from top to bottom: $2.38,4.76$, 9.52, 19.0, 38.1, 76.2, 152, 305, 609 and 1220. Solid lines represent the curves given by the present theory with the self-consistently determined parameters $\alpha_{0}=1.445$, $X=0.298$ and $q=0.413$. For the present theoretical curves (13) and (14), the number of steps in the cascade $n$ are, from top to bottom: $21,19,17,14,12,10$, 9.0, 8.0, 7.0 and 6.0. Dotted-dashed lines are the curves given by (15) for $\theta=2-q^{\prime}$. For this directly adjusted PDF, the indexes $q^{\prime}$ s depend on the distances $r / \eta$, and the adjusted values of $q^{\prime}$ are, from top to bottom: 1.215, 1.195, 1.175, 1.145, 1.115, $1.105,1.095,1.060,1.030$ and 1.005. For better visibility, each PDF is shifted by -1 unit along the vertical axis.

By making use of the point $\xi_{n}^{*}$ having only weak $n$-dependence, we divide $\hat{\Pi}^{(n)}\left(\xi_{n}\right)$ into two parts: $\hat{\Pi}^{(n)}\left(\xi_{n}\right)=\hat{\Pi}_{<*}^{(n)}\left(\xi_{n}\right)$ for $\left|\xi_{n}\right| \leq \xi_{n}^{*}$, and $\hat{\Pi}^{(n)}\left(\xi_{n}\right)=$ $\hat{\Pi}_{*<}^{(n)}\left(\xi_{n}\right)$ for $\xi_{n}^{*} \leq\left|\xi_{n}\right|$. For smaller velocity fluctuation, we assume that the PDF has Gaussian form: $\hat{\Pi}_{<*}^{(n)}\left(\xi_{n}\right)=\hat{\Pi}_{\mathrm{N}}^{(n)}\left(\xi_{n}\right)+\hat{\Pi}_{\mathrm{S}}^{(n)}\left(\left|\xi_{n}\right|\right)=\left(Z_{\mathrm{NS}}^{(n)}\right)^{-1} \mathrm{e}^{-\xi_{n}^{2} / 2 \Sigma_{n}^{2}}$, whereas, for larger velocity fluctuation, we assume that it is given by the 
distribution function for singularities [13]:

$$
\hat{\Pi}_{*<}^{(n)}\left(\xi_{n}\right)=\hat{\Pi}_{\mathrm{S}}^{(n)}\left(\left|\xi_{n}\right|\right) .
$$

Requiring $\hat{\Pi}_{<*}^{(n)}\left(\xi_{n}\right)$ and $\hat{\Pi}_{*<}^{(n)}\left(\xi_{n}\right)$ at $\xi_{n}^{*}$ to have the same value and the same derivative there, we can determine the variance $\Sigma_{n}^{2}$ and the normalization factor $Z_{\mathrm{NS}}^{(n)}$, respectively, as $\Sigma_{n}^{2}=\left(\xi^{*}\right)^{2} /\left[1+3 f^{\prime}\left(\alpha^{*}\right)\right]$ and $\left(Z_{\mathrm{NS}}^{(n)}\right)^{-1}=\bar{\Pi}_{\mathrm{S}}^{(n)} \mathrm{e}^{\left[1+3 f^{\prime}\left(\alpha^{*}\right)\right] / 2}$. Then, we see that $\hat{\Pi}_{<*}^{(n)}\left(\xi_{n}\right)$ becomes $[13]$

$$
\hat{\Pi}_{<*}^{(n)}\left(\xi_{n}\right)=\bar{\Pi}_{\mathrm{S}}^{(n)} \exp \left\{-\left[1+3 f^{\prime}\left(\alpha^{*}\right)\right]\left[\left(\xi_{n} / \xi_{n}^{*}\right)^{2}-1\right] / 2\right\} .
$$

Figs. 1 and 2 are the comparisons of the PDF $\hat{\Pi}^{(n)}\left(\xi_{n}\right),(13)$ and (14), obtained by the present theory with the experiments conducted by Lewis and Swinney [1] for $R_{\lambda}=262(\operatorname{Re}=540000)$ and $\mu=0.28$, and by Gotoh et al. [2] for $R_{\lambda}=381$ and $\mu=0.25$. In both figures, solid lines represent the curves by the present theory, whereas closed circles are the experimental data symmetrized by taking averages of the left and the right hand sides of measured PDF's. In Fig. 1 (Fig. 2), the distances $r / \eta=\ell_{n} / \eta$ are, from top to bottom: 11.6, 23.1, 46.2, $92.5208,399,830$ and 1440 (2.38, 4.76, 9.52, 19.0, 38.1, 76.2, $152,305,609$ and 1220) for the experimental data, on the other hand, the number $n$ of steps in the cascade are, from top to bottom: 14, 13, 11, 10, 9.0, 8.0, 7.5 and $7.0(21,19,17,14,12,10,9.0,8.0,7.0$ and 6.0$)$ for our theoretical lines. We observe an excellent agreement between the measured PDF and the analytical formula of PDF derived by the present self-consistent theory.

The present analysis is based on the assumptions that the distribution of the singularity indexes $\alpha$ is multifractal and that its statistics is based on the generalized measures of entropy, i.e., the Rényi entropy or the THC entropy. Note that within the present analysis we cannot distinguish which of the measures of entropies is responsible for the system of turbulence. The latter may be one of the exciting future problems both for theoreticians and for experimentalists. It is also one of the attractive future problems to find out some connection between the present ensemble approach and the one based on dynamical aspect starting with the Navier-Stokes equation, e.g., the dynamical renormalization group approach, the mode-mode coupling theoretical approach and so on.

Before closing the paper, we should mention something about the usual procedures to analyze experimentally measured PDF based on generalized statistics, e.g., Tsallis statistics which provides us with one of the possibilities for an abstract statistical mechanics for non-Gibbsian statistics that are being observed in various fields (refer to [5] for more information on recent progress). Usually, one is apt to write down Tsallis-type PDF directly without any fundamental consideration when he or she is going to analyze measured PDF. Let us explain it in the context of the present analysis of turbulence. Assuming, from 
the beginning, that the PDF of the velocity fluctuations is of Tsallis-type, one may write it in the form

$$
\hat{\Pi}_{B}\left(\xi_{n}\right) \propto\left\{1-\left(1-q^{\prime}\right)\left|\xi_{n}\right|^{2 \theta} /\left(5-3 q^{\prime}\right)\right\}^{1 /\left(1-q^{\prime}\right)}
$$

for the measured PDF symmetrized in the sense given in this paper. Here, $q^{\prime}$ is the index appearing in the Tsallis or the Rényi entropies which may have different meaning from our $q$ used in the present paper, and $\theta$ is an extra parameter determined in order to adjust (15) to the measured PDF. Note that the PDF (15) of the velocity fluctuations itself has a power-law decay for large $\delta u_{n}$, while the present PDF does not have simple power-law decay but has a bit complicated decay containing $\ln \delta u_{n}$ as can be seen in the expression (11). In Fig. 1, we put (15) with $\theta=1$ by dotted lines for comparison which is essentially the same as done by Beck in [24]. We see that this PDF cannot explain the measured one. Following the analysis done in [25], we took $\theta=2-q^{\prime}$, and put (15) in Figs. 1 and 2 for this case by dotteddashed lines just for comparison. As can be observed in Fig. 1, we cannot see big difference between our present PDF, (13) and (14), given by solid lines and the PDF, (15) with $\theta=2-q^{\prime}$, given by dotted-dashed lines, since the accuracy of experimental data for PDF was only of order $10^{-5}$. While in Fig. 2 the PDF was measured up to the order of $10^{-9}$, and we clearly see the difficulty in analyzing the data by the PDF given by Beck and others in [25]. There may be a possibility to remedy it by adjusting the dependence of $\theta$ on $q^{\prime}$ further, whose physical meaning, however, we do not know. In addition to this, the analysis based on (15) has another difficulty, i.e., the index $q^{\prime}$ depends on the distance $r / \eta$ whose dependence is listed in the captions in Figs. 1 and 2. There might exist a possible explanation about the $r / \eta$ dependence of $q^{\prime}$ by relating it to the non-extensivity of Tsallis entropy [26]. In any case, the present investigation tells us that one should choose appropriate variables before applying generalized statistics. Note that the values of $q^{\prime}$ satisfy $q^{\prime}>1$, in contrast to the values of $q$ for the present analysis where they satisfy $q<1$. It is worth mentioning here that the present ensemble theoretical approach [913 is quite general, in that the expression of the multifractal spectrum (6) based on a statistical mechanical procedure may provide an example for the phenomenological "entropy function" $S(z)$ with $z=1-\alpha$ in the problem of large deviation statistics [27]. An analysis in this direction will be reported elsewhere in the near future.

The authors would like to thank Prof. C. Tsallis and Dr. A.K. Rajagopal for their fruitful comments with encouragement, and Prof. T. Gotoh for his kindness to send them the data of his numerical simulations. 


\section{References}

[1] G.S. Lewis and H.L. Swinney, Phys. Rev. E 59, 5457 (1999).

[2] T. Gotoh, D. Fukayama and T. Nakano (2001) preprint.

[3] A. Rényi, Proc. 4th Berkeley Symp. Maths. Stat. Prob. 1, 547 (1961).

[4] C. Tsallis, J. Stat. Phys. 52, 479 (1988).

[5] C. Tsallis, Braz. J. Phys. 29, 1 (1999). On the subject see also at http://tsallis.cat.cbpf.br/biblio.htm.

[6] T. Arimitsu and N. Arimitsu, Phys. Rev. E 61, 3237 (2000).

[7] C. Meneveau and K. R. Sreenivasan, Phys. Rev. Lett. 59, 1424 (1987).

[8] C. Meneveau and K. R. Sreenivasan, Nucl. Phys. B (Proc. Suppl.) 2, 49 (1987).

[9] T. Arimitsu and N. Arimitsu, J. Phys. A: Math. Gen. 33, L235 (2000) [CORRIGENDUM: 34, 673 (2001)].

[10] T. Arimitsu and N. Arimitsu, Chaos, Solitons and Fractals 13, 479 (2002).

[11] T. Arimitsu and N. Arimitsu, Prog. Theor. Phys. 105, 355 (2001).

[12] T. Arimitsu and N. Arimitsu, Physica A 259, 177 (2001).

[13] N. Arimitsu and T. Arimitsu, cond-mat/0109132 (2001).

[14] A. M. Oboukhov, J. Fluid Mech. 13, 77 (1962).

[15] A. N. Kolmogorov, J. Fluid Mech. 13, 82 (1962).

[16] A. M. Yaglom, Sov. Phys. Dokl. 11, 26 (1966).

[17] A. N. Kolmogorov, C. R. Acad. Sci. USSR 30, 301; 538 (1941).

[18] R. Benzi, G. Paladin, G. Parisi and A. Vulpiani, J. Phys. A: Math. Gen. 17, 3521 (1984).

[19] U. Frisch, P-L. Sulem and M. Nelkin, J. Fluid Mech. 87, 719 (1978).

[20] M. L. Lyra and C. Tsallis, Phys. Rev. Lett. 80, 53 (1998).

[21] U.M.S. Costa, M.L. Lyra, A.R. Plastino and C. Tsallis, Phys. Rev. E 56, 245 (1997).

[22] A.B. Chhabra and K.R. Sreenivasan, Phys. Rev. A 43, 1114 (1991).

[23] J. H. Havrda and F. Charvat, Kybernatica 3, 30 (1967).

[24] C. Beck, Physica A 277, 115 (2000).

[25] C. Beck, G.S. Lewis and H.L. Swinney, Phys. Rev. E 63, 035303-1 (2001).

[26] C. Beck (2001) preprint.

[27] T. Watanabe, Y. Nakayama and H. Fujisaka, Phys. Rev. E 61, R1024 (2000). 